\title{
Exploration of the Meaning of A Place by Volunteered Geographic Information
}

\author{
Bor-Wen Tsai ${ }^{1, *}$, Ming-Kuang Chung ${ }^{2}$ and Yu-Ling $\mathrm{Hsu}^{3}$ \\ ${ }^{1}$ Professor, Department of Geography, National Taiwan University, Taipei, Taiwan \\ Joint-Appointment Associate Research Fellow, Academia Sinica. \\ No. 1, Sec. 4, Roosevelt Road, Taipei, 10617 Taiwan \\ ${ }^{2} \mathrm{Ph} . D$. Candidate, Department of Geography, National Taiwan University, Taipei, Taiwan. \\ No. 1, Sec. 4, Roosevelt Road, Taipei, 10617 Taiwan \\ ${ }^{3}$ Master, Department of Geography, National Taiwan University, Taipei, Taiwan. \\ No. 1, Sec. 4, Roosevelt Road, Taipei, 10617 Taiwan \\ *Corresponding author's email: tsaibw [AT] ntu.edu.tw
}

\begin{abstract}
Geographers view place as a projection of one's inner perceptions of space and is socially constructed and personally experienced. It is not merely a spatial location but something with meaning. Traditional GIS has implemented space of physical environment successfully based on computational geometry. However, how GIS can implement place which is formed through human experience from the interaction between their social activities and natural environment is still in developing stage. This research employed VGI (volunteered geographic information) concept and used geo-tagged photos from Flickr web album to delineate the meaning of a place - Gongguan. A native place located in southern part of Taipei city. The Alpha Shape, Kernel Density Estimate and Social Network Analysis were employed for analysis. Results imply that the VGI approach can be an innovative method for study of place.
\end{abstract}

Keywords - VGI, volunteered geographic information, place

\section{INTRODUCTION}

Space and place together define the nature of geography (Tuan 1979). Tuan (1975) views place as a projection of one's inner perceptions of space, the meaning of which comes from the pondering of one's place in their material environment. He considered that place is space infused with meaning (Tuan 1977). Fox (1997) considered place is socially constructed and personally experienced and space is based on directional co-ordinates and symbolic coordinates. Thomas (2001) also thought that a place is not merely a spatial location but something with meaning. Gieryn (2000) noted that place is not space, it is filled up by people, practices, objects, and representations. It is a unique space in the universe. It is not only to be delineated by geographic or cartographic metaphor such as boundary but it is also a reality to be clarified and understood from the perspectives of the people who have given it meaning. Haggett (1983) holds that space refers to a particular location, however place and location are different in that place holds humanistic value and is publically recognized as such, if a specific location becomes imbued with a deeper cultural meaning, it is then viewed as a "place". Its boundary is elastic either analytically or phenomenologically. Moreover, traditional regional geography treats location as a sort of "vessel", with this vessel being the bases of which various regional information is expounded upon, as a result simplifying the relationship between place and space (Johnston and Claval, 1984). New regional geography however focuses on collective consciousness, subjective spatial visualization and mental maps to construct the meaning of region and place. Additionally, new regional geography stresses the scope of a region is actually a "process of construction", therefore exploring the deeper meaning of the borders of place has become a principle topic in new regional geography ${ }^{1}$. For instance, studies on frontiers in the early 1990s focus not just on the material functions of borders, but also on their symbolism and latent significance. However, what remains unanswered is how can scholars

\footnotetext{
${ }^{1}$ While the term new cultural geographic has been used by scholars for some time, the meaning of the term has yet to find a clear, general consensus. Thrift, uses "reconstructed regional Geography" to refer to the recent developments in the category of Regional Geography (Thrift, 1993). Even if there is some debate around the usage of the word, there is no doubting the transformation and contribution that New Regional Geography was brought to the notions of region and place.
} 
gather collective consciousness and use it to understand the construction of space relations?

In sum, a place is located in a specific space without definite boundary and is formed through human experience from the interaction between their culture/social activities and environment. Consequently, how to depict a place from its spatial, temporal and attributive perspectives becomes the main theme of this research.

In the 21st century, a new version of World Wide Web called Web 2.0 has been developed (O'Reilly, 2006). This web application facilitates participatory and sharing information on the web. People are able to create and disseminate information instead of receiving information only. Wikipedia is probably the most well-known example. In the same time, positioning devices such as GPS (global positioning system) receivers have become civilian and popular. Many mobile devices such as smartphones, digital cameras, or tablets are GPS-embedded. Consequently, geo-tagged information can be created anytime and anywhere. Moreover, interactive Geoweb (Herring, 1994) interfaces such as Google Map, Microsoft's Bing Map or Wikimapia also make it possible for people to locate their targets of interest and disseminate their geo-tagged information. Roche et al, (2012) indicate that the merger of the interactive framework of Web 2.0 with GIS has triggered a new form of Geoweb. This Geoweb allows for both experts and citizens to access geographic information, which transforms the creation and usage of spatial data and simultaneously facilitates interactive forms of collective intelligence. Together with this development, there has been a growing interest to create and share geographic information on the web by individuals. Goodchild (2007) has termed "volunteered geographic information (VGI)". It has caught increasing attention by geographers for VGI changes the way geographic data are collected, compiled, distributed, and exploited. Turner (2006) has called it "neogeography" which is about sharing location information with friends and visitors, helping shape context, and conveying understanding through knowledge of place.

This paper presents a VGI approach by utilizing geo-tagged photos from Flickr web album to explore the meaning of a native place. Tagged photos are at their essence a form of social tagging which reflect the public's general notions of said environment. Moreover, photo tags reveal users' actions, behaviours, and the role that people, situations, time, land, and objects play in the creation of space. The native place of interest is Gongguan located in southern part of Taipei City. Alpha Shape, Kernel Density Estimate (KDE) and social network analysis (SNA) are employed to identify Gongguan's boundary and content. Temporal analysis is also conducted to investigate the revolutionary character of place.

\section{THE VGI APPROACH TO EXPLORE A PLACE}

VGI is amateur but professional (Goodchild 2007; Flanagin and Metzger 2008). Many research issues have been raised such as data credibility, validity and authority (Elwood 2008a, 2008b; Flanagin and Metzger 2008) and its assessment methods (Bishr and Mantelas 2008; Coleman, et al. 2009; Gouveia and Fonseca 2008; Raykar, et al. 2010; Dickenson, et al. 2010). Contributors' motivation (Coleman et al. 2010, Goodchild 2007 and Elwood, 2008b) and the societal impact of VGI are other issues that got great attention. However, the most valuable contribution of VGI is its innovative outcome. Elwood (2008b) argued that VGI might enable new forms of knowledge. Recent research (Deng et al., 2009; Grothe and Schaab, 2009), demonstrated that geotags reflect the ways in which individuals view a particular location, and through the analysis of this sort of collective intelligence, we can define the true extent of informal place and regions. Among VGI data, web photo album is one of the most popular data sources used in VGI research. Sigurbjörnsson and Zwol (2008) analyzed tags of 52 million Flickr photos and found 28\% of tags depict location information. Wilske (2008) used photos from Flickr, Panoramio and Locr web albums to delineate the boundary of Upper West Side in New York City by employing the egg-yolk theory. Grothe and Schaab (2009) developed an automated method of footprint and its boundary generation based on the Kernel Density Estimation and support vector machines (SVM). Martins (2011) delimited imprecise regions with Flickr photos and land cover data, with results showing that the proposed method performs better than previous studies that used web photos only. However, these studies focused on the spatial aspect of photos such as the distribution pattern or boundary generation by geotagged photos. None of the studies pay attention to attributive aspect such as the context of a place.

With respect to photo content, Jiang (2013) considered VGI has enormous potential for studying spaces and places. Elwood, et al. (2013) considered VGI tends to inherit the character of human discourse rather than scientific measurement. They raised the concept of "the place-based GIS" (platial GIS). Capinri (2016) considers VGI consists three essential components, where, what and who. The what records contributor's points of view, values, feelings, expression and represents the 'sense of place'. It deals with areas which are typically not defined by administrative boundaries such as 'neighborhood' or 'down down' which are often considered to be vague. Adams and McKenize (2013) considered a sense of place is the result of subjective experience that a person has from being a place or from interacting with information about a place. They used topics modeling (a natural language processing) to identify latent topics in a large corpus of travel blog entries that describe places around the world and examining how the topics are distributed over space and time. The rationale behinds Adams and McKenize's method is that people choose to write about a place in the blog reflects their sense of that place, and by generalizing over many people's writing, one can extrapolate an aggregate view of that place. Bischoff, et al. (2010) indicated that photo tags can comment and express opinions subjectively on a resource based on social motivation. In sum, existing research has shown the usefulness of geotags and tags of web photos or social media to study the physical and social perspectives of a place. 


\section{THE TARGET PLACE OF INTEREST}

Gongguan is a native place in southern part of Taipei City and originally an immigrant settlement in 1730s. After the Taihoku Imperial University (the National Taiwan University's institutional predecessor) was founded in 1928 by the Japanese colonial administration, it became a living area of university's students and staffs. Nowadays, it is a landmark of Taipei City (Figure 1). This research does not intend to explore the place of Gongguan before 20th century because of data limitation. Instead, this research tries to use VGI data to explore the meaning of a place and its change in modern era.

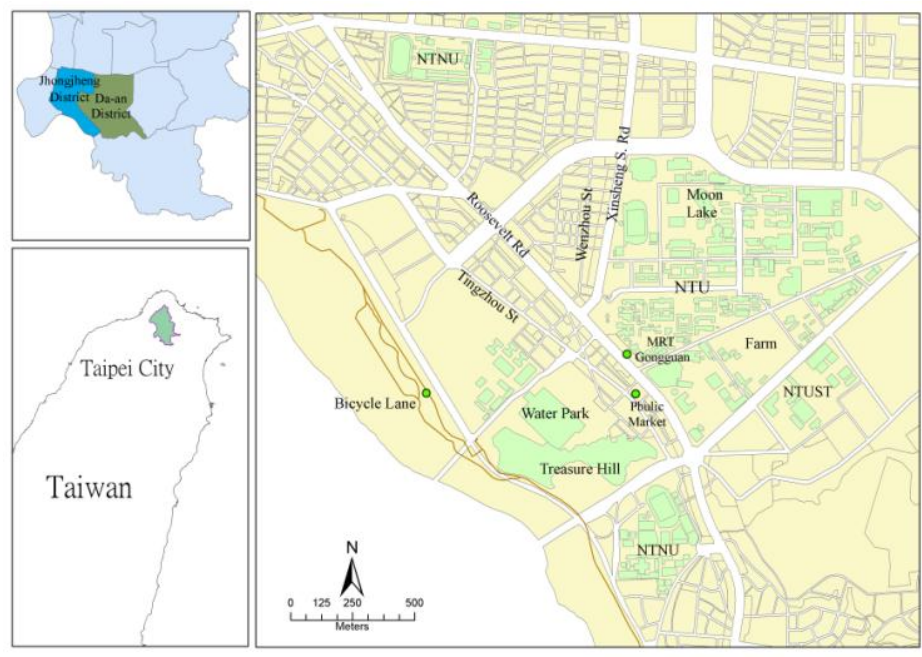

Figure 1: The Study Area

\section{MATERIALS}

Flickr is one of the most commonly used data sources of VGI. According to 2013 statistics, Flickr owns almost 12 million users, accounting for $70 \%$ of total internet user population in Taiwan (Yahoo Inc., 2015). In terms of location information, Flickr uses the GPS location of where the photo was taken, or the user, when uploading the photo, selects its location on an interactive maps. Facebook and Instagram's location determination uses a Location Base Service (LBS) instead, which selects an existing geotag near by the user to determine location. In comparison, Flickr data possess more accurate location information than other social media data.

Web photos tagged with "公館", "Gung gong", "gong guan" or "gongguan" were extracted from flicker web album. A total of 1,777 photos were collected dated from January, 2004 to July, 2013 (Table 1). These photos were screened out based on: 1) without geotags; 2) not located in Taipei City (same name in other prefectures); and 3) duplicate photos. Of the 1,777 photos, 527 photos were without geotags or not in Taipei City. The remaining 1,250 photos were contributed by 261 volunteers. Photos taken by the same contributors at the same time were excluded to avoid domination by few contributors . Consequently, 595 photos were analyzed in this research.

Table 1. Total Photos and Qualified Photos

\begin{tabular}{ccc}
\hline Year & Qualified Photos & Total Photos \\
\hline 2004 & 4 & 5 \\
\hline 2005 & 6 & 5 \\
\hline 2006 & 23 & 31 \\
\hline 2007 & 36 & 30 \\
\hline 2008 & 85 & 239 \\
\hline 2009 & 205 & 440 \\
\hline 2010 & 102 & 417 \\
\hline 2011 & 81 & 278 \\
\hline 2012 & 40 & 300 \\
\hline 2013 & 13 & 32 \\
\hline Sum & 595 & 1,777 \\
\hline
\end{tabular}

\section{METHODS}

The Alpha Shapes (Edelsbrunner, et al. 1983) is generated from point sets to form the conceptual region of Gongguan. It represents an enclosed region of all geo-tagged photos that tagged with Gongguan. However, spatial variation exists in 
the region. In order to investigate the distribution density within the region, Kernel Density Estimate (Diggle 1985) is further calculated to address the density surface of Gongguan region. The Alpha Shape is primarily based on the dispersal of the data points as opposed to their quantity. It can mitigate the influence of outliers to some extent by defining a proper search radius (Cope, 2008). Kernel Density Estimate is able to demonstrate where the data clustered. However, it is susceptible to the influence of duplicate data points by one dominant photo contributor (Grothe and Schaab, 2009). Consequently, we employs both methods complementary to identify the boundary and the clusters within the region.

The Alpha Shape is generalization of the concept of the convex hull. For a set of points $\mathrm{S}$, a point $\mathrm{p}$ in $\mathrm{S}$ is said to be a boundary node if $\mathrm{p}$ lies on the boundary of a closed disc of radius $\alpha$ that contains no other points of S. Connecting all such points $1, \mathrm{p} 2, \ldots$ and pn forms an Alpha Shape of S (Figure 2). If $\alpha-->\infty$, then the Alpha Shape of S is its convex hull. If $\alpha->0$, then the Alpha Shape will becomes negative shape (Figure 3). If $\alpha=0$, the Alpha Shape degenerates to the point-set $S$.

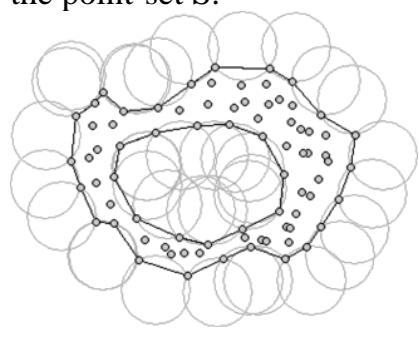

Figure 2: Alpha Shape

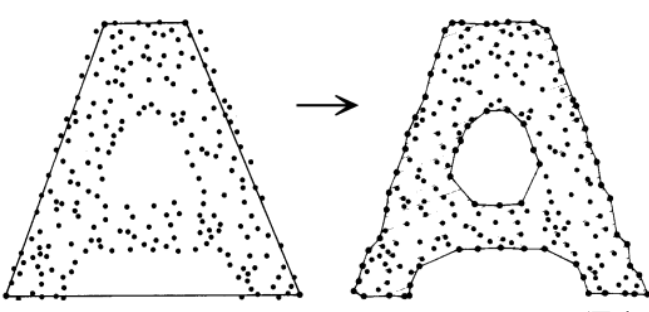

(Edelsbrunner et al.,1983)

Figure 3: Positive shape and negative shape

The Kernel Density Estimate is defined as:

$$
f(x)=\frac{1}{n} \sum_{i=1}^{n} K_{h}\left(x-X_{i}\right)
$$

where

$\mathrm{f}(\mathrm{x})$ : Kernel Density, n: number of points, h: search radius, K: Kernel Function, x: vector at point x, Xi: vector at point Xi.

In addition to analyze the conceptual region of Gongguan by photos' spatial tags. The Social Network Analysis (Scott 2006) is used to explore how people cognize Gongguan by photos' tags. These tags can be viewed as user's generated meta data (Mathes, 2004), and is the act of assigning key words to assist in the process of categorization. Social Network Analysis views social relationships in terms of network theory. In this research, nodes represent individual tag contents, and ties represent relationships between the individual tag content. A network graph is used to depict the network. The eigenvector centrality index was calculated to identify the important or influenced nodes within the network. The Microsoft Excel open-source template NodeXL (http://nodexl.codeplex.com/) is used to generate the network graph and the index calculation.

Put together all three methods, the Alpha Shape and Kernel Density Estimate are used to explore where people think about Gongguan, while the social network analysis is used to explore how people think about Gongguan.

\section{RESULTS AND DISCUSSION}

The Alpha Shape and Kernel Density surface for 2004-2008 and 2009-2013 are generated and shown in Figure 4. The first-level tags identified by the social network analysis are shown in Figure 5 and Table 2. The first-level tags indicate the most important nodes in the network. However, there are tags that were attached to photos automatically when photos were uploaded to the web album. These tags include names of administration areas such as TAIWAN and TAIPEI and information for the photo such as NIKON, FILM, BW (black and white photo) and so on. These tags appear in almost every photo and can be identified as the most important nodes. However, they do not imply any image of Gogguan and are not included in the following interpretation. 

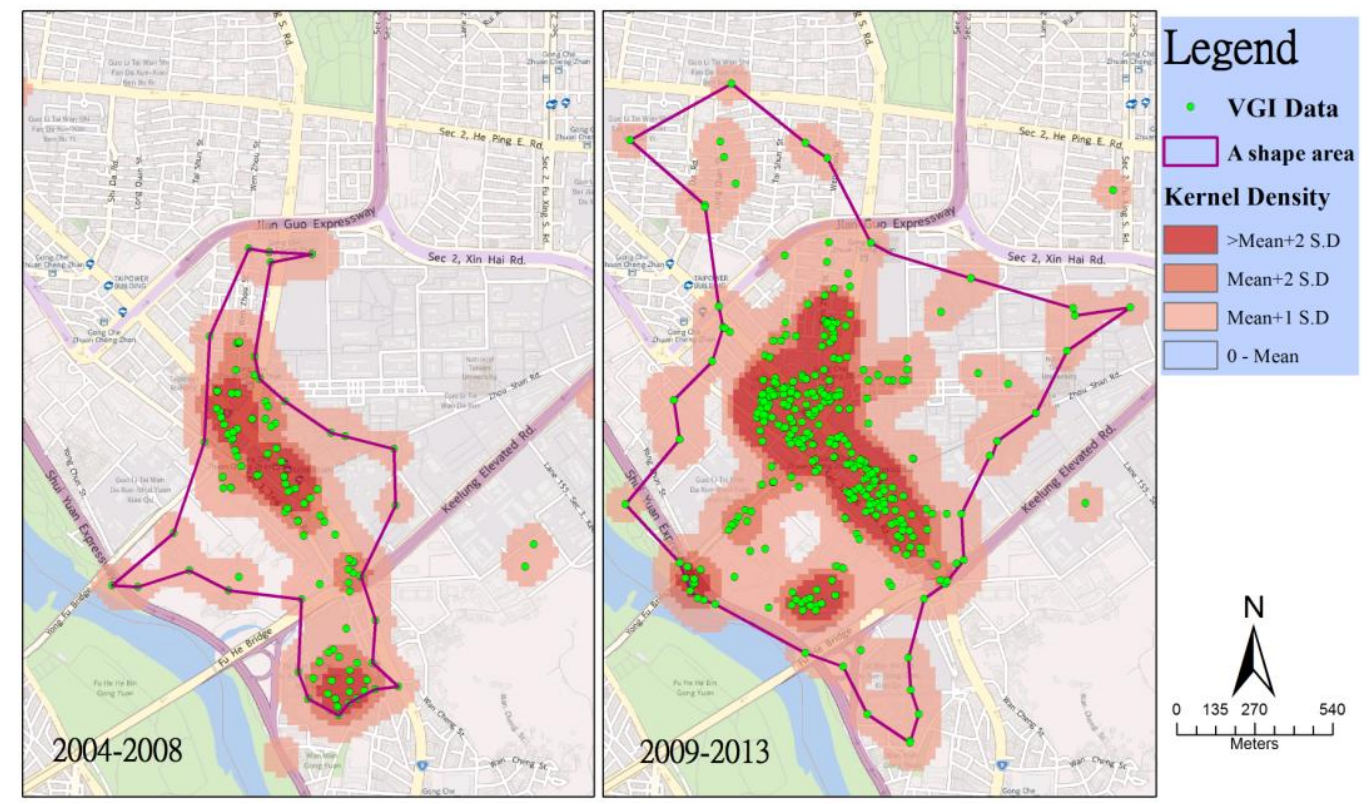

Figure 4 Conceptual regions and density surface of Gongguan

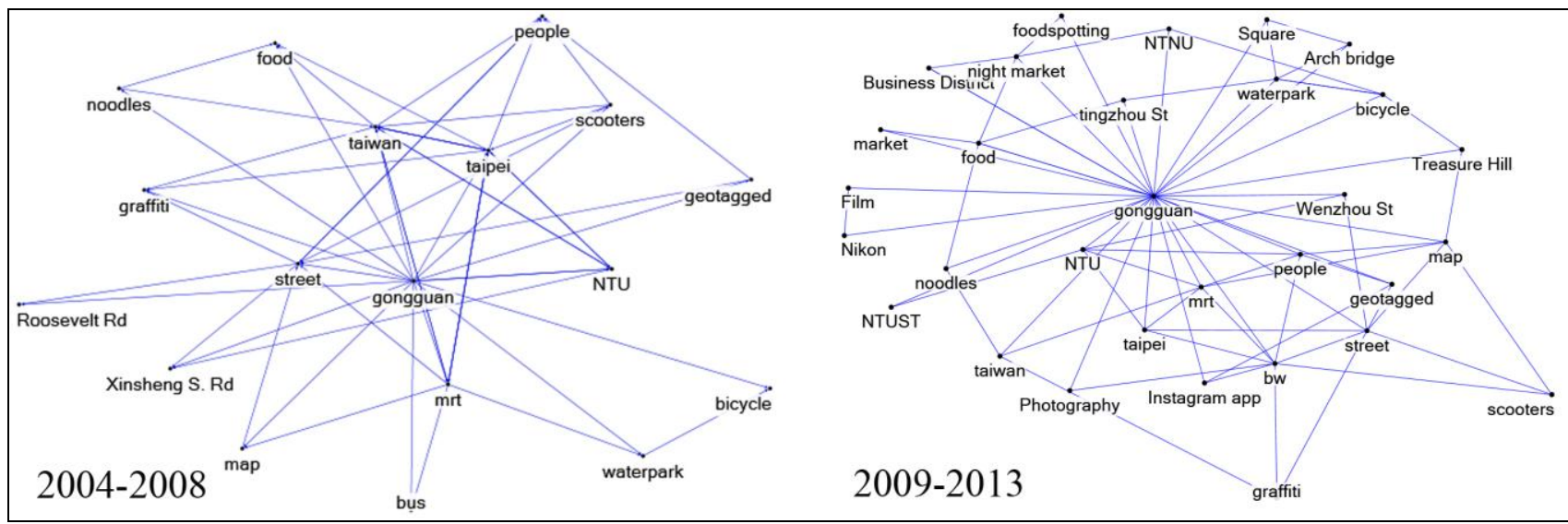

Figure 5 First-level Network Graph of Gongguan by NodeXL 
Table 2. The 1st-Level Tags and Their Centrality Index.

\begin{tabular}{|c|c|c|c|}
\hline & Tag & $\begin{array}{c}\text { Eigenvector } \\
\text { Centrality } \\
(2004-2008)\end{array}$ & $\begin{array}{c}\text { Eigenvector } \\
\text { Centrality } \\
(2009-2013)\end{array}$ \\
\hline \multirow{9}{*}{ Location } & NTU (National Taiwan University) & 0.050 & 0.042 \\
\hline & map & 0.042 & 0.042 \\
\hline & Roosevelt Rd. & 0.030 & - \\
\hline & Xinsheng S. Rd. & 0.027 & - \\
\hline & Wenzhou St. & - & 0.028 \\
\hline & NTNU (National Taiwan Normal University) & - & 0.024 \\
\hline & $\begin{array}{l}\text { NTUST (National Taiwan University of Science } \\
\text { and Technology) }\end{array}$ & - & 0.022 \\
\hline & business district & - & 0.020 \\
\hline & Tingzhou St. & - & 0.024 \\
\hline \multirow{4}{*}{ Transportation } & street & 0.074 & 0.049 \\
\hline & mrt & 0.074 & 0.051 \\
\hline & scooters & 0.066 & 0.020 \\
\hline & bus & 0.030 & - \\
\hline \multirow{13}{*}{ Leisure } & graffiti & 0.058 & 0.018 \\
\hline & people & 0.055 & 0.047 \\
\hline & food & 0.053 & 0.029 \\
\hline & noodles & 0.041 & 0.024 \\
\hline & Water Park & 0.034 & 0.029 \\
\hline & bicycle & 0.024 & 0.027 \\
\hline & night market & - & 0.029 \\
\hline & Treasure Hill & - & 0.025 \\
\hline & photography & - & 0.023 \\
\hline & arch bridge & - & 0.023 \\
\hline & square & - & 0.023 \\
\hline & market & - & 0.020 \\
\hline & food spotting & - & 0.020 \\
\hline \multirow{8}{*}{$\begin{array}{l}\text { Automatically } \\
\text { attached tags }\end{array}$} & Taiwan & 0.093 & 0.029 \\
\hline & Taipei & 0.087 & 0.043 \\
\hline & Gongguan & 0.125 & 0.116 \\
\hline & geotagged & 0.039 & 0.033 \\
\hline & Nikon & - & 0.019 \\
\hline & Film & - & 0.019 \\
\hline & $\mathrm{BW}$ & - & 0.054 \\
\hline & Instagram app & - & 0.028 \\
\hline
\end{tabular}

For the conceptual region of Gongguan, the 2004-2008 region was mainly along Roosevelt Road which is a major north-south avenue in Taipei City. Consequently, this area became a transportation hub in southern part of Taipei City. City residents, especial younger generation, visited the region frequently because of convenient transportation . Restaurants and shops were the major land use types in the region. This conceptual region delineated by the Alpha Shapes and Kernel Density surfaces can be verified by the results of social network analysis. STREET, MRT, SCOOTERS, BUS of the transportation-related tags and GRAFFITI, PEOPLE, FOOD, NODDLE, WATER PARK and BICYCLE of the leisure-related tags are shown in the first-level social network graph (Figure 5, Table 2). Consequently, transportation and leisure activities were the major image impressed by people at this stage. The location-related tags of first-level tags including NTU (National Taiwan University), ROOSEVELT RD and XINSHENG RD also coincide with the corresponding region depicted by Alpha Shapes and Kernel Density surfaces. In sum, the conceptual region of Gongguan was recognized along the Roosevelt Road and its nearby areas and cognized as a place of transportation and leisure at the stage of 2004-2008.

A couple of significant changes in the conceptual region can be seen between 2004-2008 and 2009-2013. First, the Alpha Shapes show significant expansion to the north, northeast and southwest sides of the Roosevelt Road. Second, the Kernel Density shows an expanded and intensified kernel along the Roosevelt Road. Last, there are three emerging isolated kernels and one disappeared kernel (Figure 6). It is obvious that the conceptual region of Gongguan expands. It can be verified by the location-related tags such as WENZHOU ST and TINGZHOU ST and leisure-related tags such as TREASURE HILL, NIGHT MARKET, WATER PARK, BICYCLE, ARCH BRIDGE and so on. For the image of 
Gongguan, there are twelve new tags appearing and three tags disappearing from the first-level tags. In general, five new location-related tags contribute to the interpretation of region expansion by Alpha Shapes analysis, while seven new leisure-related tags contribute to the interpretation of emerging kernels by Kernel Density Estimate. The transportationrelated tags remain unchanged except the BUS tag. It is speculated that buses were no longer the most popular mode of transport after the completion of the MRT system. However, Gongguan was still cognized as a transportation hub. In sum, the conceptual region of Gongguan was expanded from the period of 2004-2008 to 2009-2013. The kernel along Roosevelt Road is intensified. Three new kernels emerge and one disappears. For the image of Gongguan, it was still congnized as a place of transportation and leisure at this stage.

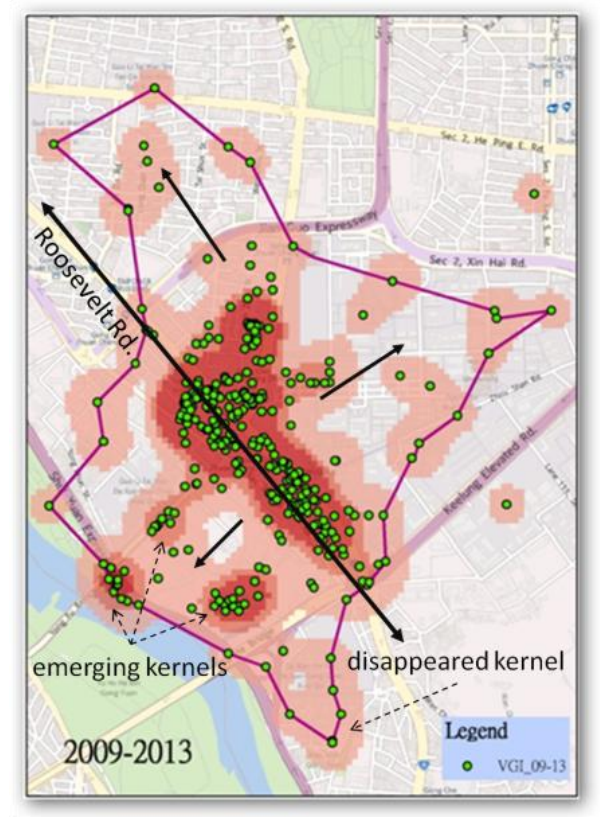

Figure 6 Expansion of Gongguan

This research shows that Alpha Shape can be used to delineate a boundary from dispersed point data. The region bounded by this boundary can be seen as an extent of the place of Gongguan. However, a place is a space without definite boundary. Kernel Density Estimate is able to show different levels of concentration within the boundary of Gongguan. This capability is coincident with the definition of place by the new regional geography. In addition to the boundary or extent, people's impression is the main theme to explore a place. The social network analysis is a way to identify the most impressed views by visitors.

The VGI and the analysis methods used in this research are distinct from traditional methods of questionnaire or interview in three perspectives. First, data (web photos) are not produced intentionally but built in daily activities and reflect personal experience. On the contrary, data collected by questionnaire or interview are answers on the fly and usually influenced by atmosphere or scenario of the survey. Second, most positions indicated in web photos are generated automatically by mobile devices, whereas positions from questionnaire or interview are usually translated from verbal description and easy to cause errors. Last, as It is a difficult or error-prone process to memorize activities in the past, photos are the best media to record activities. Consequently, web photos are the suitable material for temporal analysis.

\section{CONCLUSION AND FUTURE WORK}

Researchers usually use questionnaire and interview to explore the meaning of a place. This study demonstrates that VGI is an alternative method. Critical issues for VGI include crowd data extraction, data analysis and interpretation. This study employs Alpha Shape, Kernel Density Estimate and Social Network Analysis for data analysis. The former two are used to generate the conceptual region and the latter the place's image.

New regional geographers consider a region without a definite boundary, with such a region being subjectively formed and a dynamic process. Consequently, the boundary of such a region will change from time to time. The research results show that the extent of Gongguan has expanded during two time periods. However, the image of Gongguan remains unchanged, It is recognized as a place for living activity instead of historical or cultural context.

This research demonstrates the usefulness of VGI and related analysis methods to study a place. However, computer illiteracy issue is the major concern when VGI data are the only data source for analysis. Integration of different data sources can be considered. The limited (595) number of photos available at the time of study is another defect. However, It does not affect the main theme of this paper that demonstrating a VGI approach to study a place. 


\section{REFERENCES}

[1] Adams, B. and McKenize, G., "Inferring thematic places from spatially referenced natural language descriptions", In Crowdsourcing Geographic Knowledge, edited by D. Sui, S. Elwood and M. Goodchild, pp. 201-222, Springer, Dordrecht, 2013.

[2] Bischoff, K., Firan, C.S., Nejdl, W. and Paiu, R., "Bridging the gap between tagging and querying vocabularies: Analyses and applications for enhancing multimedia IR", Journal of Web Semantics, vol. 8, no.2-3, pp. 97-109, 2010.

[3] Bishr, M. and Mantelas, L., "A trust and reputation model for filtering and classifying knowledge about urban growth”, GeoJournal, vol. 72, pp. 229-237, 2008.

[4] Capineri, C., "The nature of volunteered geographic information", in Capineri, C. Haklay, M., Huang, H., Antoniou, V., Kettunen, J., Ostermann, F. and Purves, R. (eds), European Handbook of Crowdsourced Geographic Information, Ubiquity Press Ltd, London, 2016.

[5] Coleman, D., Sabone, B. and Nkhwanana, N.J., "Volunteering geographic information to authoritative databases: Linking contributor motivations to program characteristics", Geomatica, vol. 64, no. 1, pp. 27-39, 2010.

[6] Coleman, D., Georgiandou, Y. and Labont, J., "Volunteered geographic information: The nature and motivation of produsers", International Journal of Spatial Data Infrastructures Research, vol. 4, pp. 332-358, 2009.

[7] Diggle, P.J., "A kernel method for smoothing point process data”, Journal of the Royal Statistical Society C, vol. 34, pp. 138-147, 1985.

[8] Dickenson, J.L., Zuckerberg, B. and Bonter, D.N., "Citizen science as an ecological research tool: Challenges and benefits", Annual Review of Ecology, Evolution, and Systematics, vol. 41, pp. 149-172, 2010.

[9] Elwood, S., "Volunteered geographic information: key questions, concepts and methods to guide emerging research and practice", GeoJournal, vol. 72, pp. 133-135, 2008a.

[10] Elwood, S., "Volunteered geographic information: Future research directions motivated by critical, participatory and feminist GIS", GeoJournal, vol. 72, pp. 173-183, 2008b.

[11]Elwood, S., Goodchild, M.F. and Sui, D., "Prospects for VGI research and the emerging fourth paradigm", In Crowdsourcing Geographic Knowledge, edited by D. Sui, S. Elwood and M. Goodchild, pp.361-375, Springer, Dordrecht, 2013.

[12] Edelsbrunner, H., Kirkpatrick, D.G. and Seidel, R., "On the shape of a set of points in the plane", IEEE Trans Inform. Theory IT, vol. 29, no. 4, pp. 551-559, 1983.

[13] Flanagin, A.J. and Metzger, M.J., "The credibility of volunteered geographic information”, GeoJournal, vol. 72, pp. 137-148, 2008.

[14]Fox, J.J., "Genealogy and Topogeny", In The Poetic Power of Place: Comparative Perspectives on Austronesian Ideas of Locality, edited by J.J. Fox, Canberra, The Australian National University, 1997.

[15] Gieryn, T.F., “A space for place in sociology”, Annual Review of Sociology, vol. 26, pp. 463-496, 2000.

[16] Gilbert, A., "The new regional geography in English and French-speaking countries", Progress in Human Geography, vol. 12, no. 2, pp. 208-228, 1988.

[17] Goodchild, M.F., "Citizens as sensors: The world of volunteered geography”, GeoJournal, vol. 69, pp. 211-21, 2007.

[18] Gouveia, C. and Fonesca, A., "New approaches to environmental monitoring: The use of ICT to explore volunteered geographic information", GeoJournal, vol. 72, pp. 185-197, 2008.

[19] Grothe, C. and Schaab, J., "Automated footprint generation from geotags with kernel density estimation and support vector machines", Spatial Cognition and Computation, vol. 9, pp.195-211, 2009.

[20]Herrying, Charles, " An architecture for cyberspace: Spatialization of the Internet", U.S. Army Construction Engineering Research Laboratory, 1994.

[21] Jiang, B., "Volunteered geographic information and computational geography: New perspectives", In Crowdsourcing Geographic Knowledge, edited by D. Sui, S. Elwood and M. Goodchild, pp. 125-138, Springer, Dordrecht, 2013.

[22] Lou, Shan, Jiang, Xiang and Scott, Paul J., "Geometric computation theory for morphological filtering on freeform surfaces", Proceedings of the Royal Society A: Mathematical, Physical and Engineering Sciences, 2013.

[23] Martins, B., "Delimiting imprecise regions with georeferenced photos and land coverage data", In 10th International Symposium, W2GIS 2011, In Lecture Notes in Computer Science, edited by K. Tanaka, P. Fröhlich and K.S. Kim, 6574, pp. 219-229. Springer-Verlag, Berlin Heidelberg, 2011.

[24] Miller, C., "A beast in the field: The Google Maps mashup as GIS”, Cartographica, vol. 41, pp.1878-1899, 2006.

[25] Negoescu, R.A. and Gatica-Perez, D., "Analyzing Flickr groups", In Proceedings of the 2008 International Conference on Content-Based Image and Video Retrieval, pp. 417-426, ACM, Niagara Falls, Canada, 2008.

[26] Newman, D., "Fences and neighbours in the postmodern world: Boundary narratives in political geography", Progress in Human Geography, vol. 22, no. 2, pp. 186-207, 1998.

[27] Paasi, A., "Boundaries as social practice and discourse: The Finnish-Russian border", Regional Studies, vol. 33, no. 7, pp. 669-680, 1999.

[28] Raykar, V.C., Yu, S., Zhao, L.H., Valadez, G.H., Florin, C., Bogoni, L. and Moy, L., "Learning from crowds." Journal of Machine Learning Research, vol. 11, pp. 1297-1322, 2010. 
[29] Sigurbjörnsson, B. and Zwol, R.V., "Flickr tag recommendation based on collective knowledge", Proceeding of the 17th International Conference on World Wide Web, ACM, Beijing, China, pp. 327-336, 2008.

[30] Scott, J., Social Network Analysis: A Handbook, Sege Publications Inc, London, 2006.

[31] Thomas, J., “Archaeologies of place and landscape”, In Archaeological Theory Today, edited by I. Hodder, Policy Press, Cambridge, 2001.

[32] Tuan, Y.F., Landscapes of Fear, Pantheon Books, New York, 1979.

[33] Turner, A., Introduction to Neogeography, O’Reilly Media, Sebastopol, CA, 2006.

[34] Williams, S., “Application for GIS specialist meeting." Accessed November $20,2015$. http://www.ncgia.ucsb.edu/projects/vgi/docs/position/Williams_paper.pdf, 2007.

[35] Wilske, F. “Approximation of neighborhood boundaries using collaborative tagging systems", In GI-Days 2008, edited by Pebesma, E., Bishr, M., Bartoschek, Germany: Institute for Geoinformatics, 2008. 